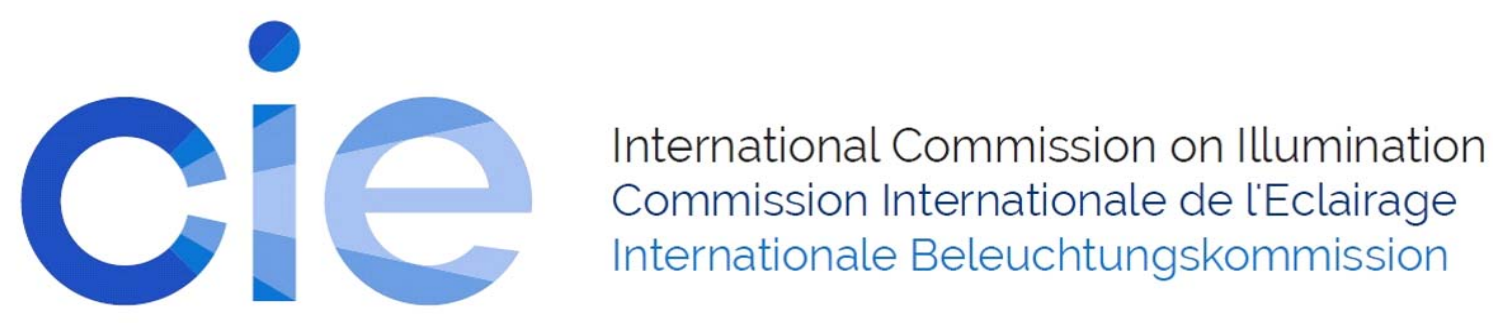

OP51

FAILING MECHANISMS OF LED LAMPS

Hans Baumgartner et al.

DOI 10.25039/x46.2019.OP51

from

CIE x046:2019

Proceedings

of the

29th CIE SESSION

Washington D.C., USA, June 14 - 22, 2019

(DOI 10.25039/x46.2019)

The paper has been presented at the 29th CIE Session, Washington D.C., USA, June 14-22, 2019. It has not been peer-reviewed by CIE.

(C) CIE 2019

All rights reserved. Unless otherwise specified, no part of this publication may be reproduced or utilized in any form or by any means, electronic or mechanical, including photocopying and microfilm, without permission in writing from CIE Central Bureau at the address below. Any mention of organizations or products does not imply endorsement by the CIE.

This paper is made available open access for individual use. However, in all other cases all rights are reserved unless explicit permission is sought from and given by the CIE.

CIE Central Bureau

Babenbergerstrasse 9

A-1010 Vienna

Austria

Tel.: +43 17143187

e-mail: ciecb@cie.co.at

www.cie.co.at 


\title{
FAILING MECHANISMS OF LED LAMPS
}

\author{
Baumgartner, H. ${ }^{1}$, Vaskuri, A. ${ }^{1}$, Kärhä, P. ${ }^{1}$, Ikonen, E. ${ }^{1,2}$ \\ ${ }^{1}$ Aalto University, Espoo, FINLAND, \\ ${ }^{2}$ VTT Technical Research Centre of Finland Ltd., Espoo, FINLAND \\ hans.baumgartner@aalto.fi
}

DOI 10.25039/x46.2019.OP51

\begin{abstract}
We have aged 20 specimens of commercial LED lamps for 62000 hours at typical room conditions since 2011. After 62000 hours of continuous operation, the luminous fluxes of 9 specimens out of 20 were still above $70 \%$ of the initial value. During ageing, the luminous fluxes of 5 specimens decreased below $70 \%$ and 6 specimens failed. The failing of the broken lamps was studied by disassembling the housing and examining the LEDs and their driving electronics. In cases when the lamp broke, the reason for the failure was the failing of the driving electronics. Overheating of the power electronics and broken electrolytic capacitors were found by examining the circuit boards. In addition to failed electronics, severe physical damages on the LED chips were found. In some LED types, the dome covering the semiconductor chip had cracked and in some LED types the luminophore coating had become transparent, which decreased the luminous flux of the LED. Characterization of the broken LEDs revealed high leakage currents and unstable current-voltage-characteristics.
\end{abstract}

Keywords: ageing, failing, lamps, LED, lifetime

\section{Introduction}

Solid state lighting based on light-emitting diodes (LEDs) has substituted incandescent light sources in most applications in recent years. Illuminating Engineering Society of North America (IESNA) has defined the lifetime of LED lamps by lumen maintenance, and the lamp is at the end of its lifetime when the luminous flux has decreased to $70 \%$ of its initial value (IESNA 2011). The expected lifetime of LED lamps is typically more than 25000 hours, corresponding to 2.85 years of continuous operation. Natural ageing of LED lamps over such a long period is time consuming and thus little objective scientific research has been published on the real lifetimes of LED lamps. Typically ageing tests to project the lifetime LED light sources are carried out at elevated temperatures to accelerate the ageing of the samples thus shortening the ageing time required. However, validating the lifetime projections is not possible without knowledge of the real lifetime in room conditions. (Baumgartner 2016)

In our ageing study, we have aged 20 specimens of commercial LED lamps for 62000 hours to study the real lifetime of LED light sources. To obtain statistical data, the ageing study included four specimens of each lamp type. The failed lamp specimens were disassembled to study the failing mechanisms of the electronics and LED chips. To the authors' knowledge, a lifetime research based on such a long ageing period has not been published before.

\section{Samples and ageing setup}

To study the lifetimes, five different types of LED lamps from Philips and Osram were purchased. High quality manufacturers were chosen, because it was assumed that the electronics of cheap lamps might fail during ageing and defeat the study. The electrical power and manufacturer specifications for the expected lifetimes of the studied lamps are presented in Table 1. 
Table 1 - Manufacturer's specifications of the five different types of LED lamps studied.

\begin{tabular}{|c|c|c|}
\hline & Power [W] & Lifetime [h] \\
\hline Type A & 12 & 25000 \\
\hline Type B & 4.5 & 35000 \\
\hline Type C & 8 & 25000 \\
\hline Type D & 12 & 25000 \\
\hline Type E & 12 & 25000 \\
\hline
\end{tabular}

All the lamp types used a switched mode power supply and a separate printed circuit board for the LEDs. All the lamps had an E27 socket and they were driven with $230 \mathrm{~V}$ AC voltage. Instead of a single circuit board, lamp type A had three separate boards at the angles of $120^{\circ}$ for the high power LEDs. The currents driving the LEDs were initially measured by disassembling the lamps (not the specimens to be aged) and measuring the current waveforms inside the lamps. The LEDs of the lamp types A, D, and E were driven with DC currents of $205 \mathrm{~mA}, 370 \mathrm{~mA}$ and $380 \mathrm{~mA}$, respectively. The LEDs inside the lamp types $B$ and $C$ were driven with pulse width modulated currents with peak currents of $790 \mathrm{~mA}$ and $840 \mathrm{~mA}$, respectively.

The lamp specimens were aged at room temperature and measured periodically for spectral radiant flux, luminous flux, electrical power, and luminous efficacy. Before the first measurement round, the lamps were pre-aged for 100 hours to stabilize the lamp electronics and the LEDs.

\section{Measurement setup}

The lamps were measured in a 1.65-m integrating sphere for the relative spectral radiant flux (4m geometry, $\lambda=360-830 \mathrm{~nm}, \Delta \lambda=1 \mathrm{~nm}$ ), luminous flux, electrical power, and luminous efficacy. The luminous flux signal produced by the LED lamp was measured with a photometer, the photocurrent of which was measured using a current-to-voltage converter and a multimeter. The luminous flux responsivity of the integrating sphere photometer is traceable to the detector based illuminance scale of National Metrology Institute of Finland (Hovila 2004, Poikonen 2012). For relative spectral radiant flux measurement, a calibrated spectroradiometer with a diffuser head was used in the detector port of the sphere. The spectroradiometer was calibrated against a spectral irradiance standard lamp with traceability to the spectral irradiance scale of MRI (Kübarsepp 2000). Figure 1 shows a photograph and schematics of the integrating sphere measurement setup used to carry out the measurements.
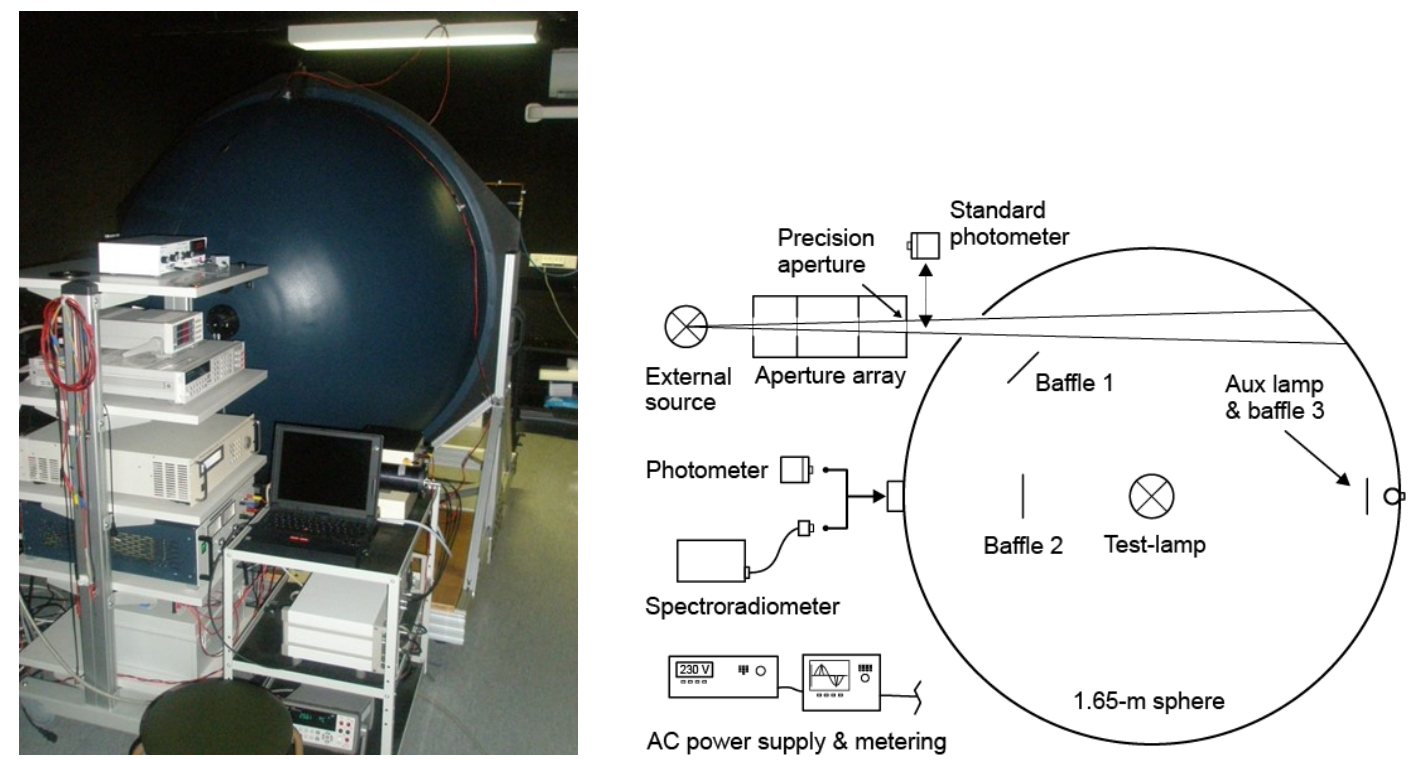

Figure 1 - Integrating sphere setup used to measure the spectral radiant flux, luminous flux, electrical power, and luminous efficacy of the studied LED lamps. 


\section{Measurement results and failing mechanisms}

The correlated colour temperature calculated form the spectral radiant flux measurements changed less than 5\% during the first 50000 hours of continuous operation of all lamp specimens. Due to the relatively small changes in spectral features of the lamps, the change of spectra are not included in the lifetime analysis. Figure 2 shows the average changes for the luminous flux and luminous efficacy for the lamps aged. As seen in the figure, the luminous efficacy follows the luminous flux of the lamps, indicating a constant electrical power consumption and decrease in luminous flux.
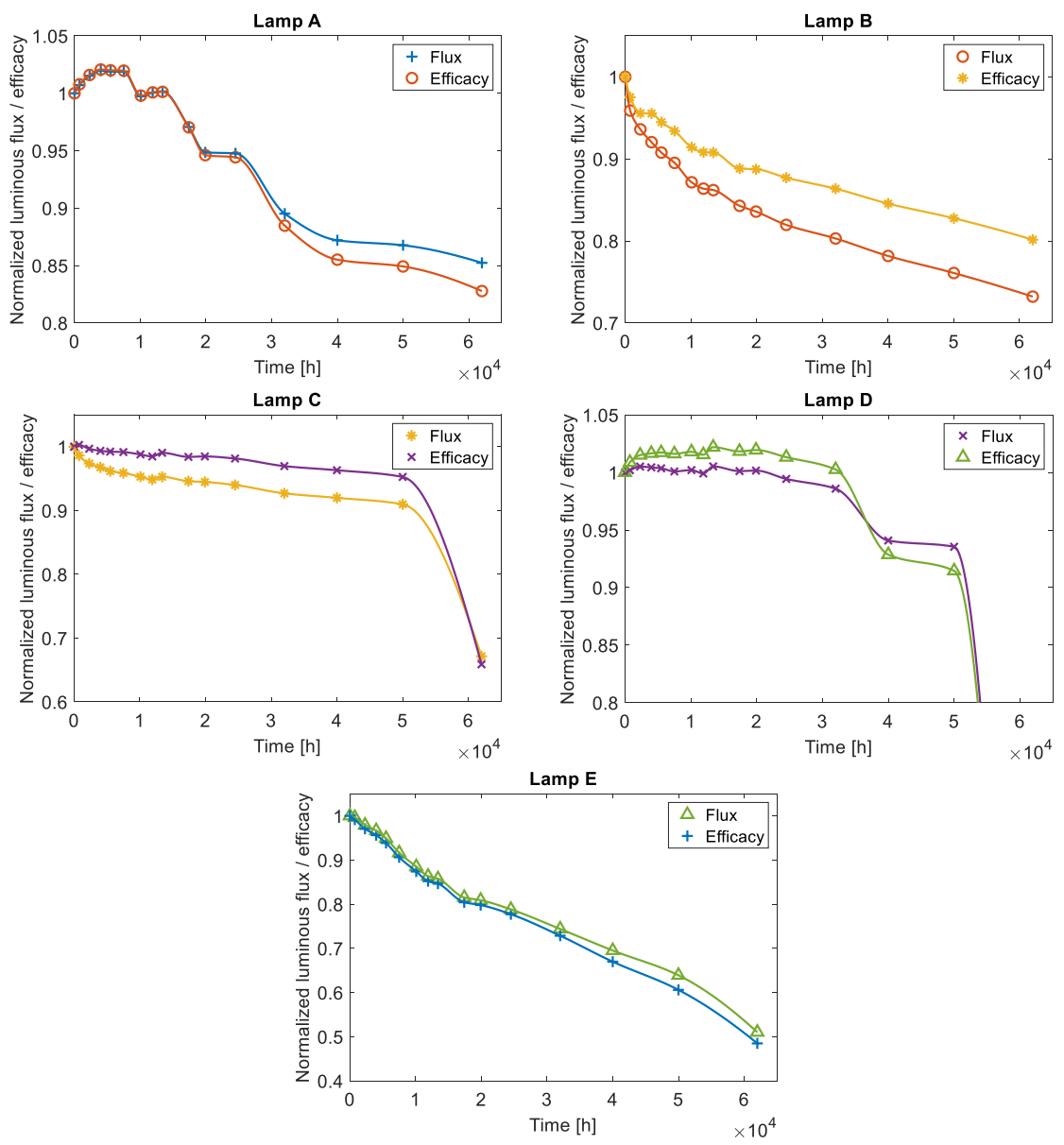

Figure 2 - Average changes of luminous flux and luminous efficacy as a function of ageing time in hours.

IESNA has defined the LED lamp lifetime through the lumen maintenance in IES TM-28-14. The expected lifetimes for the LED lamp specimens in our study have been projected earlier using the ageing data of 24500 hours (Baumgartner 2016). The lifetime projections were based on using exponential fits to the ageing data and extrapolating the measurement results to the time when the luminous flux will decrease below $70 \%$ of the initial value.

Using the ageing data between 12000 hours and 24500 hours, the projected lifetimes were 60900 hours, 353000 hours, and 39300 hours for the lamp types B, C, and E, respectively. For the lamp types $A$ and $D$, the lifetimes could not be projected due to the non-decreasing luminous flux during the ageing. As seen in Figure 2, the real lifetime of the lamp type $B$ was little more than 60000 hours as predicted. The lifetime of lamp type $C$ was less than 60000 hours. Compared to the predicted value of 353000 hours, the early projection did not predict the correct lifetime. This is due to the slow decrease of the luminous during ag eing and sudden drop of the luminous flux after 50000 operating hours. The real lifetime of lamp type $E$ was 40000 hours which is near the projected value of 39300 hours. As seen from the ageing results, the exponential fit to project the expected lifetime of LED lamps is suitable for some LED lamp types, but does not give reliable lifetime projections for all types of LED lamps. 
In addition to the broken lamp specimens of type D and E, a single specimen of type A failed as well after 62000 operating hours. The failing mechanisms of the broken lamp specimens were studied by disassembling the broken lamp specimens of types $A, D$, and $E$.

The LEDs in lamp type A were assembled on three separate circuit boards with six LEDs on each. The LEDs of the broken specimen were all operational but the power supply had failed. No visible damage was found on the power supply circuit board. In addition to the broken lamp specimen, a single LED in one type A lamp was found to be broken after 7500 operating hours. Figure 3 shows photographs of the three sides of lamp A specimen with a single broken LED.
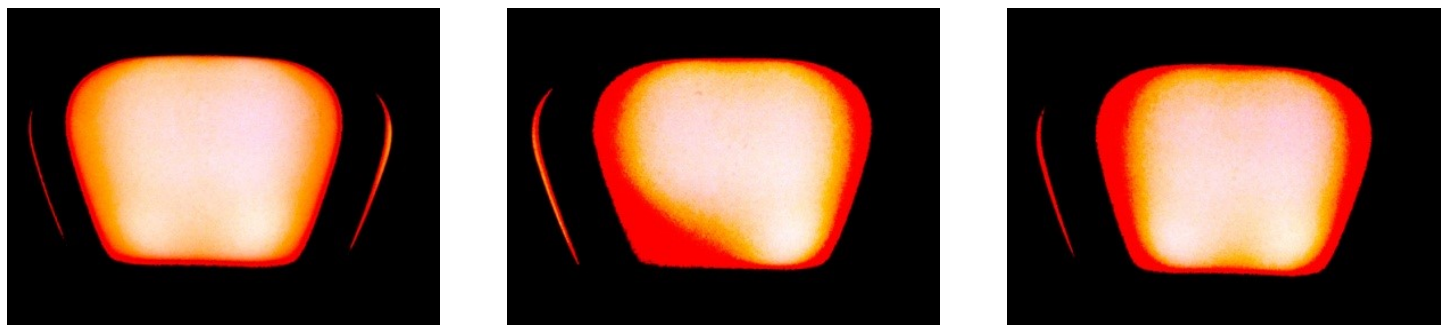

Figure 3 - Photographs of the three sides of a lamp A specimen where a single LED has failed.

For the three broken specimens of lamp type $D$, the failing was found to be due to failed power supply. In addition to the broken power supply, electrically and mechanically broken LEDs were found as well. The lamp type D comprised red and white LEDs to produce warm light with a correlated colour temperature of $2700 \mathrm{~K}$. The LEDs were characterized for current-voltagecharacteristics using a source measure unit. The domes of the broken white LEDs had visible cracks, but there were no mechanical damage in the red LEDs. Figure 4 shows a photograph and the I-V curve for broken and not broken white and red LEDs of inside a broken lamp of type D.
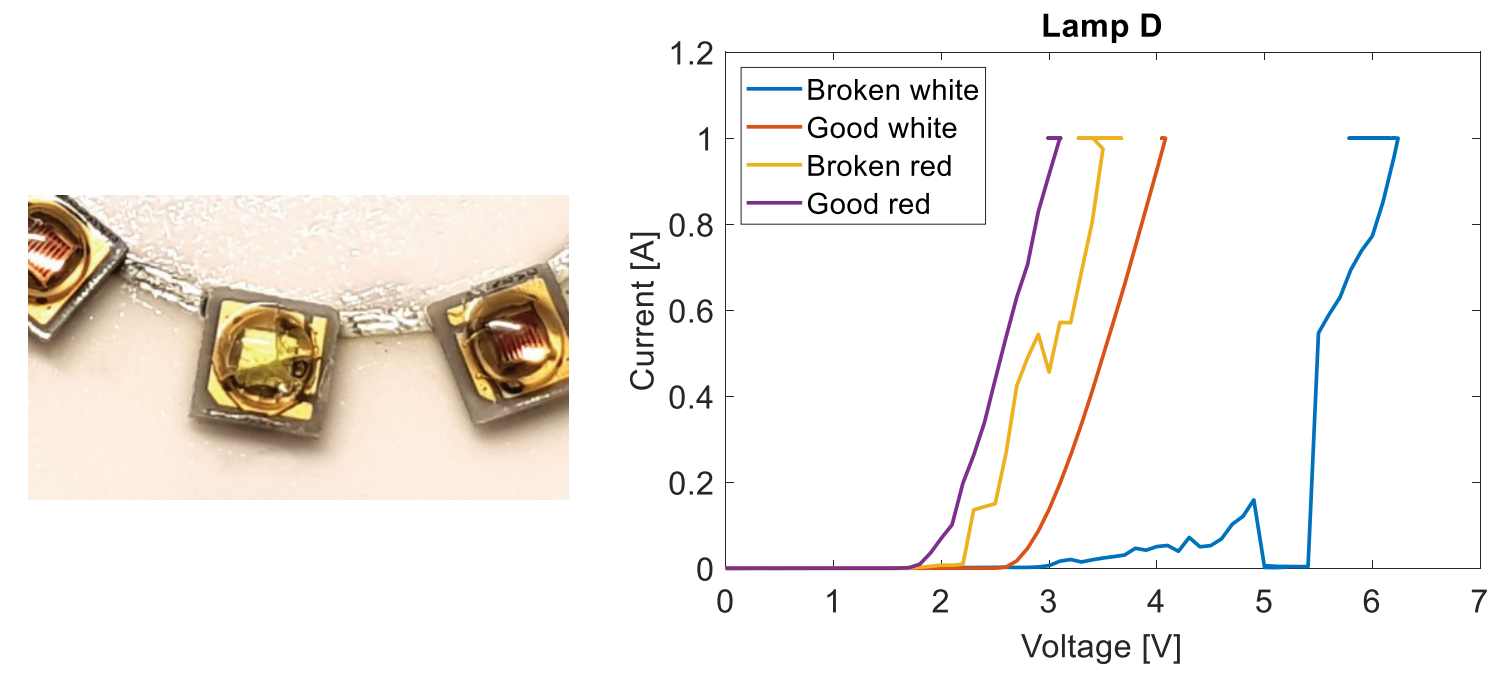

Figure 4 - Photograph of a broken white LED (cracks) and I-V characteristics for the LEDs inside lamp type $D$.

As seen in Figure 4, some LEDs in a lamp with broken power supply were damaged as well. The damage in the LEDs led to increased forward voltage and unstable I-V characteristics. Due to increased forward voltage, more power are required from the power supply to provide constant current, which may have affected the lifetime of the power supply electronics. In both lamps types $D$ and $E$, similar power supply design was used. By opening the housings of lamp types D and E, severely overheated power transistors and broken electrolytic DC capacitors were found from the power supplies. Figure 5 shows a broken and overheated power supply from lamp type $D$. 


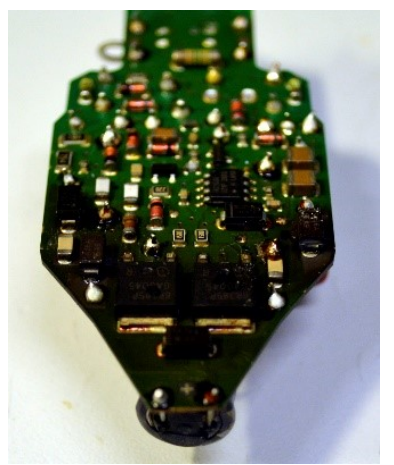

Figure 5 - Severely overheated power transistors inside the power supply of lamp type D were found.

The broken lamp type $\mathrm{E}$ was studied for failing mechanisms by disassembling the lamp housing. In the two broken lamp specimens of type $E$, the power supplies were failed. In addition to the broken power supply, the luminophore coating of LEDs had become transparent with brownish shade as shown in Figure 6 . The changes in the luminophore may be the main reason for the fast decrease of luminous flux of lamp type E. The LED circuit board of lamp type E comprise nine white LEDs, the I-V characteristics of which were analysed. Several LEDs were failed leading to high leakage current and resistor-like current-voltage-characteristics as shown in Figure 6.
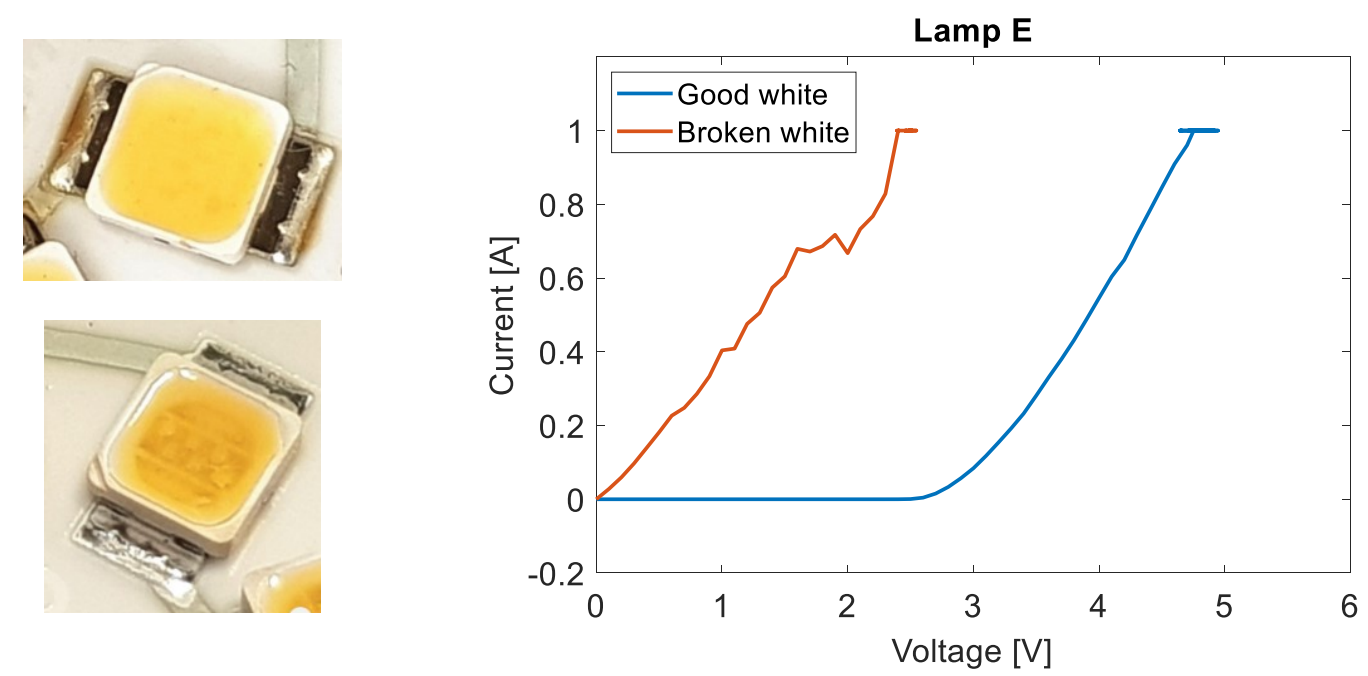

\section{Figure 6 - Photographs of a new (upper) and a broken (lower) white LEDs and I-V} characteristics of broken and good white LED inside lamp type $E$.

\section{Conclusions}

Five different types of LED lamps were aged for 62000 hours in normal room conditions to study their real lifetime. The expected lifetimes of LED lamps are tens of thousands of hours requiring ageing periods of several years to achieve their real lifetimes. We have aged five different types of LED lamps as long as their luminous fluxes decrease below $70 \%$ of the initial value, which is the lifetime definition of LED lamps by IESNA. Our results show that the real lifetimes of the lamp types studied are longer than the manufacturer lifetime specifications. In all cases when the lamp failed, the power supply had failed due to the overheating of the power electronics and failing of the electrolytic capacitors. In addition to the failed power supply severe damage in the LEDs were found as well.

In some LED types, the dome covering the semiconductor chip had cracked and in some LED types, the luminophore coating had become transparent, which decreased the luminous flux of the LED. The current-voltage-characteristics of the broken LEDs were analysed as well, revealing high leakage currents and unstable operation of the LEDs. 


\section{References}

IESNA 2011. IES TM-21-11. Projecting Long Term Lumen Maintenance of LED Packages. New York.

BAUMGARTNER, H. et. al. 2016. Natural and accelerated ageing of LED lamps. Lighting Res. Technl. 48, 930-942.

HOVILA, J. et. al. 2004. Realization of the unit of luminous flux at the HUT using the absolute integrating-sphere method. Metrologia 41, 407-413.

KÛBARSEPP T. et. al. 2000. Spectral irradiance measurements of tungsten lamps with filter radiometers in the spectral range $290-900 \mathrm{~nm}$. Metrologia 37, 305-312.

POIOKNEN T. et. al. 2012. Luminous efficacy measurement of solid state lamps. Metrologia 49, S135-140. 